\title{
La recherche à l'université de Poitiers de 2004 à
} 2007

\section{(2) OpenEdition Journals}

Édition électronique

URL : http://journals.openedition.org/abpo/252

DOI : $10.4000 /$ abpo. 252

ISBN : 978-2-7535-1512-3

ISSN : 2108-6443

\section{Éditeur}

Presses universitaires de Rennes

\section{Édition imprimée}

Date de publication : 31 décembre 2008

Pagination : $333-340$

ISBN : 978-2-7535-0808-8

ISSN : 0399-0826

\section{Référence électronique}

"La recherche à l'université de Poitiers de 2004 à 2007 », Annales de Bretagne et des Pays de l'Ouest [En ligne], 115-4 | 2008, mis en ligne le 31 décembre 2008, consulté le 14 septembre 2020. URL : http:// journals.openedition.org/abpo/252

Ce document a été généré automatiquement le 14 septembre 2020.

(c) Presses universitaires de Rennes 


\section{La recherche à l'université de Poitiers de 2004 à 2007}

1 Liste des thèses et mémoires soutenus et déposés à l'université de Poitiers.

\section{BIBLIOGRAPHIE}

\section{Master 1}

\section{4}

AUDOUIN, Laure, Les dernières volontés des Montmorillonnais. Approches et mentalités religieuses des habitants du «pays » montmorillonnais face à la mort de 1650 à 1792 à travers l'acte testamentaire, dir. F. VIGIER.

BELCH ESQUINA, Simon, La table médiévale : discriminations sociales et représentations mentales dans les œuvres littéraires (fin XIe-XVe siècle), dir. M. AURELL.

BENHIDA, Nadia, La gestion de l'anormalité dans l'asile de Beauport à Québec de 1845 à 1893, dir. F. CHAUVAUD.

BERNARD, Aline, Les imprimés révolutionnaires du département de la Vienne (1789-1799) conservés à la Médiathèque François Mitterrand de Poitiers et aux Archives Départementales de la Vienne, dir. F. VIGIER. BILLY, Maud, L'univers des cabarets et auberges de la sénéchaussée de Montmorillon au XVIIIe siècle, dir. F. VIGIER.

CASTEX, Anthony, A la rencontre du Bouddhisme. La perception du Bouddhisme chez les voyageurs et géographes aux XVIIe et XVIIIe siècles, dir. S. JAHAN. 
DEFONTAINE, Émilie, Etre perruquier à Poitiers sous l'Ancien Régime. Étude économique, sociale et culturelle des perruquiers de Poitiers, de la fin du XVIIe siècle à la veille de la Révolution, dir. J. PÉRET. DELMAS, Raphaëlle, Justice et religion au siège royal de Lusignan de 1701 à 1758, dir. F. VIGIER.

DIOT, Ameline, Louis Guillemot, Alexandre et François Guillemot. Correspondance entre trois frères sous la Révolution et l'Empire, dir. F. VIGIER.

DOUANIER, Marie-Carole, Les femmes et la mort dans les faits divers de l'entre deux guerres (à partir des exemples des journaux l'Avenir de la Vienne et le Journal de l'Ouest), dir. F. CHAUVAUD.

DUPONT, Olivier, La vienne et le recrutement militaire de l'an VIII à 1808, dir. N. PETITEAU EDNA, Pierre, Le mouvement indien équatorien dans les années 1990, dir. A. LEMPÉRIÈRE. FLEURIAUD, Geoffrey, Le vol au village. Vols et voleurs dans les colonnes de l'Avenir de la Vienne et du Journal de l'Ouest entre 1870 et 1914, dir. F. CHAUVAUD.

FLEURY, Louise, Etre femme de député poitevin à l'époque révolutionnaire, dir. ANNE JOLLET.

FOURNAISE, Bruno, Les Plantagenêt et le Berry dans la seconde moitié du XIIe siècle, dir. M. AURELL.

FRANÇOIS, Olivier, Aliénés et aliénistes. Les maladies de l'esprit : l'idiotie, la démence, la manie, la paralysie générale... à travers le Dictionnaire encyclopédique des sciences médicales Dechambre, dir. F. CHAUVAUD. GALENON, Sophie, Une vision française du contentieux franco-britannique en 1942 : l'exemple de l'affaire de Diégo-Suarez, dir. G. BOURGEOIS.

GLATIGNY, Jessica, Les faits divers liés au milieu maritime d'après L'Evangéline (1900-1930), dir. F. CHAUVAUD.

GODENECHE, Pierre, Essai sur une forme de lutte dans une ville moyenne de province: des manifestations de rue à Poitiers en 1986, 1995, 1997, dir. S. коTT.

GREAU, Virginie, Les espaces criminels dans la presse régionale à travers l'exemple de La Nouvelle République du Centre-Ouest de 1945 à nos jours, dir. F. CHAUVAUD.

JOFFRET, Marie, Coups, horions, blessures... La violence et ses représentations dans un quotidien régional, l'Avenir de la Vienne (1919-1939) (2 vol.), dir. F. CHAUVAUD.

KHUN, Nida, En quête de l'identité criminelle. Des empreintes et des signes dans Le traité de criminalistique (1931-1940) d'Edmond Locard, dir. F. CHAUVAUD.

LOPEZ Navarro, Hélène, Les relations Mère-fille en Grèce Ancienne : les tragédies grecques ou la figure des talaina (2 vol.), dir. L. BODIOU.

MASSE, Julien, Etre gréviste dans la Vienne des années 20 (1919-1932), dir. F. CHAUVAUD.

MAUSSET, Hélène, Chaînes, relégués et transportés des pavés de La Rochelle à l'enfer de Cayenne de 1873 à 1938. Le bagne transit de Saint-Martin-de-Ré, dir. F. CHAUVAUD.

MEILHAC, Cécile, Les commémorations acadiennes du début du xxe siècle à aujourd'hui sur le Web, dir. D. GUILLEMET.

MICHEL, Aurore, La religiosité dans la France du bas Moyen-Age : d'après les deux inventaires de mobiliers de Charles V de 1363 et 1380, dir. E. BоZоку.

PARBAILLE, Jérôme, Conception et exercice de la piété dans le Pythagorisme ancien : VIe-IVe siècles av. J.-C., dir. Y. LAFOND. 
PONS, Nicolas, Le rôle de la papauté dans la constitution des lieux de pèlerinage romain du pontificat de Damase (366-384) à celui de Grégoire IV (827-844), dir. E. воZоKY.

RIVAUD, Armandine, Les élections municipales dans la Vienne en 1977, dir. P. LÉVY.

ROUSSEAU, Virginie, Les artisans du bois de Poitiers au XVIIIe siècle. Essai d'analyse professionnelle, économique, sociale et culturelle

ROUYER, Aline, Mélusine au XVe siècle : du mythe à la réalité, dir. I. MARCHESIN.

SYOEN, Arnaud, Mémoires et aménagement urbain dans le quartier de St-Cybard à Angoulème, dir. S. KOTT.

THEVENET, Emmanuel, L'hôpital général de Poitiers à l'âge classique, 1659-1789 (2 vols), dir. F. VIGIER. TRANCHANT, Aurélie, Chauvigny au fil des voies publiques : les enjeux de leur dénomination, dir. F. CHAUVAUD.

ZEEGERS, Marieke, Approche informatisée de l'image sigillaire de la femme sur les sceaux féminins : de 1150 à 1350 (3 vol.), dir. M. AURELL.

ZIANI, Kamal, La vie politique au Maroc de 1965 à 1973 vue à travers le journal Le Monde, (2 vol.), dir. S. MICHONNEAU.

2007

BAILLARGEAU, Estelle, La scolarisation des filles au primaire au Burkina Faso depuis l'indépendance (1960-2005) (2 volumes), dir. F. CHAUVAUD.

BAUDOIN, Cécile, Les tisserands et les tailleurs d'habits de Poitiers au XVIIIe siècle. Étude économique, sociale et culturelle des tisserands et des tailleurs d'habits poitevins de 1703 à 1792.

BERTRAND, Bruno, La vision occidentale du tatouage aux îles Marquises : de l'arrivée de James Cook à la prise de possession par l'amiral Dupetit-Thouars, dir. S. JAHAN.

BOUTET, Ludovic, Les migrants saisonniers du pays thouarsais dans la paroisse d'Arçay au XVIIIe siècle, dir. S. JAHAN.

BUREAU, Amandine, Les veuves et leurs enfants dans la diaspora acadienne (1755-1840) (2 vol.), dir. D. GUILLEMET et Th. SAUZEAU.

CAILlaud, Sarah, Captivités, tortures et adoptions chez les Iroquois de l'Amérique du Nord-Est (1641-1667): Étude comparée de 3 récits de captivités, dir. D. GUILLEMET et S. JAHAN.

CARPENTIER, Julie, L'image de l'Amérindien dans les correspondances militaires pendant la Guerre de Sept Ans, dir. D. GUILLEMET et S. JAHAN.

CAULIER, Étienne, L'épée de connétable (2 vol.), dir. M. AURELL.

CHANCEREL, François, Buxerolles à la fin de l'Ancien Régime, dir. D. GUILLEMET et J. PÉRET.

CHATELIER, Guillaume, L'impact de la Révolution française sur le marché ordinaire : étude comparative des années 1788 et 1793 dans la région de Poitiers (2 vol.), dir. A. JOLLET.

COUEDEL, Laetitia, La famille dans la matière de Bretagne au XIIe siècle, dir. M. AURELL.

COURTOT-AUDOUSSET, Elisabeth, Une ville en fête : les commémorations de Jeanne d'Arc à Poitiers, leur représentation dans la presse poitevine de la fin du XIXe siècle à 1945, dir. F. CHAUVAUD. 
COUTURIER, Cédric, La violence aux États-Unis dans la presse française pendant l'entre deux guerres, dir. F. CHAUVAUD.

DAMY, Jeanne, Albert Londres et André Gide au cœur des années 20 : Regards croisés sur l'Afrique Noire française, dir. F. CHAUVAUD.

DE CORBIER, Guilhem, Les nobles protestants du Poitou pendant les Guerres de religion (1562-1598), dir. D. TURREL.

DEMICHEL, Fanny, La prostitution à Poitiers au XVIIIe siècle dans les faubourgs de Montierneuf et Saint Hilaire (1685-1789), dir. S. JAHAN.

FREGE, Coralie, La cour métropolitaine et la cour primatiale de Poitiers pendant la 1re moitié du XVIIIe siècle, dir. F. VIGIER.

GREGOIRE, Audrey, L'émigration espagnole dans le département de la Vienne 1808-1833, dir. N. PETITEAU. GROS, Samuel, Les samouraï vus par les premiers explorateurs européens au Japon, du XVIe au début de l'ère Meiji, dir. S. JAHAN.

GUERIN, Marie, La Morée franque XIIIe-XVe siècle : les francs et la société grecque à travers la chronique de Morée, dir. Е. воZоку.

HUGUE, Jennifer, Étude d'une partie de la bibliothèque de Gui Bernard évêque duc de Langres et paire de France (1453-1481) : huit manuscrits conservés à la Médiathèque François Mitterrand de Poitiers, dir. C. TREFFORT.

LAVAUR, Ludivine, Ecrire ses dernières volontés en Poitou aux XVIIe et XVIIIe siècles 2 vol., dir. F. VIGIER. LEBEDEL, Gaëlle, Les aubergistes du Poitou au Cour d'une société révolutionnaire, dir. F. VIGIER. LEROUX, Caroline, Prénoms, délais au baptême et conceptions à Lusignan, Chauvigny et Mirebeau dans la seconde moitié du XVIIe siècle, dir. F. VIGIER.

LIGNER, Florian, Évolution de l'image de l'Islande dans les guides touristiques (1950-2003), dir. F. CHAUVAUD.

LUSSEAU, Sylvain, César géographe, d'après ses Commentaires de la Guerre des Gaules, dir. J. HIERNARD. MAGNIN, Magali, Le souvenir de l'Empire et de Napoléon dans le département de la Vienne sous La Restauration (1815-1830), dir. N. PETITEAU.

METAYER, Aurélie, Le culte de Tyché dans les cités de Grèce continentale aux époques hellénistique et romaine, dir. Y. LAFOND.

MICHAUD, Sandrine, Auberges, cabarets et leurs tenanciers dans le Montmorillonnais et le Limousin de 1718 à 1775. «In vino veritas » : sociabilités et conflictuosités dans les débits de vin, selon les minutes de greffe de la sénéchaussée de Montmorillon, dir. F. VIGIER.

PANOU, Julien, Le suicide et la presse régionale dans le département de la Vienne 1919-1938.

Роснот, Benjamin, Les vacances et le tourisme en Roumanie (1964-1974).

PRINCAY, Caroline, Les artisans du fer à Poitiers au XVIIIe siècle, dir. J. PÉRET.

PUAUD, Olivier, La compagnie de l'île Saint-Jean (1719-1724), dir. D. GUILLEMET et T. SAUZEAU.

TALLEUX, Maryline, Jumeaux : les naissances multiples en Poitou au XVIIIe siècle, dir. S. JAHAN.

TAUNAY, Arnaud, Étude socio démographique de la population châtelleraudaise au XIXe siècle, dir. N. PETITEAU. 
VERMOGEN, Amélie, Naissances et prénomination dans les villes de Châtellerault, Montmorillon, Mirebeau, Lusignan, Chauvigny et Civray de 1793 à l'an VII, dir. F. VIGIER.

WISSOCQ, David, Poitiers, la nuit. Une ville de province en quête de sa modernité nocturne (1870-1930), dir. F. CHAUVAUD.

\section{6}

BERNARD, Aurélien, A-t-on voulu relever la Nouvelle France? La politique de soutien aux colonies par le port de Rochefort au début de la paix de 30 ans, vue à travers la correspondance ministérielle, dir. Th. SAUZEAU.

BERRY, Mélanie, Le Baron de Montmorency : Une entreprise, des voyages et des hommes : 1773-1782 : Un navire rochelais à la fin du dix-huitième siècle, dir. T. SAUZEAU.

BOTTON, Claire, Mariage et Famille dans le Haut-Bocage dans la seconde moitié du XVIIIe siècle. D'après le contrat de mariage passé autour de Pouzauges entre 1742 et 1784.

CHAMPALOU, Aurélie, Les boulangers à Poitiers au XVIIIe siècle. Étude professionnelle, sociale, culturelle et économique.

CHANTRE, Luc, La République et le Hajj (1880-1931). Le pouvoir colonial et le pèlerinage à La Mecque.

GEAHEL, Edith, La représentation des enfants dans la presse satirique de la Belle Époque. L'exemple du Rire 1894-1914.

GIRARDEAU, Tristan, Les sensibilités religieuses du " pays » de Gâtine entre 1660 et 1789. Approches de la religiosité des habitants de Parthenay et sa région à partir des actes testamentaires.

GRIMAUD, Noémie, Les sociabilités villageoises dans le civraisien de 1850 à 1950. Fêtes et Solidarités Paysannes

GRIPPON, Adeline, Demandeurs d'asile aux portes de l'Europe : l'exemple de la Slovénie et de la Maison d'Asile de Ljubljana, dir. F. CHAUVAUD.

HA, Seolin, L'image de la Chine, de la Corée et du Japon en Europe : premiers contacts.

JEAN, Samuel, Les litiges liés aux activités rurales et au travail dans les jugements civils de la justice de paix de Pleumartin 1844-1885.

KRAUSKOPF, Charles-Alexandre, Une sensibilité provinciale face à un évènement de portée nationale : l'exemple de la commémoration de la Commune de Paris à Poitiers (1871-1914).

LEGRAND, Séverine, Les exécutions capitales en France de 1880 à 1939 à travers le Journal de l'Ouest.

LOUIS, Thomas-Pierre, L'économie de la guerre de course rochefortaise sous Louis XIV, pendant la guerre de la Ligue d'Augsbourg (1688-1697).

LUCAS, Sophie, Cheltenham entre 1815 et 1852 : La création d'un Espace urbain raffiné.

MORISSET, Romain, L'intégration européenne de l'Islande (1957-2006).

Moumbouli, Sandrine, Des Criminelles et des Femmes : Le crime féminin de 1919-1939 d'après le Journal de l'Ouest.

NAULLEAU, Anne-Clémentine, Les faits divers dans les Affiches du Poitou (1773-1781).

PIRA, Fanny, Les femmes criminelles dans le film noir américain de 1941 à 1955.

SAFFROY, Ludovic, Violences et déviances nocturnes dans la Vienne (1850-1900) 
SEGALAT, Marie, La médecine féminine au XVIIIe siècle : Guérir les corps et les esprits, choisir les mots et les remèdes : Dans le traité des maladies des femmes de Jean Astruc, dir. S. JAHAN.

VERGNAULT, Antoine, Le commerce maritime à Rochefort durant la guerre d'Indépendance américaine : activité des hommes et des navires entre 1778 et 1783. D'après l'étude des rôles de désarmement, ainsi que des registres de matricules des navires et des gens de mer principalement, dir. T. SAUZEAU.

WIMART, Stéphane, D'un rivage à l'autre, Rochefort et l'île Royale durant la Paix de Trente Ans (1716-1744). Le rôle de l'arsenal dans la fondation, la militarisation et l'approvisionnement de la colonie à partir des dépêches ministérielles, dir. T. SAUZEAU.

\section{7}

ACHARD, Hélène, La promenade en image à la Belle Époque : d'après l'Assiette au Beurre et L'Illustration : Journal Universel.

ARAFELOFF, Sophie, Les fêtes révolutionnaires à Poitiers (1789-1799).

AUDOUIT, David, Les Royannais sous les bombes. La vie quotidienne de la population civile royannaise pendant le siège de la forteresse de Gironde-Nord (12 septembre 1944-17 avril 1945).

BEDOURET, Julie, «Sang » elles à la « Une ». Fémicides et quotidiens régionaux l'Avenir de la Vienne et le Journal de l'Ouest, 1912-1914/1918-1925.

BERNARD, Amélie, Harry Haase, prisonnier de guerre allemand. Itinéraire d'un P.G.A., manœuvre agricole à Mazières-sur-Béronne de 1945 à 1948.

BLANCHET, Aurélie, De la Charente au Mississipi, Rochefort et la Louisiane de Rémonville à la Rétrocession (1710-1730).

BONNET, Claire, Ecrire ses maux. La femme et sa santé dans la noblesse française du XVIIIe à travers les lettres des duchesses de Chaulnes et de Gontaut Biron.

BOUFFARD, Anne-Sophie, La publicité dans le Journal de l'Ouest durant la période de l'entre-deux-guerres. BRANCHU, Alexandre, Les gens de mer royannais au XIXe siècle (1824-1883).

BULAM, Sarban, Léodile Béra, observatrice sociale (1824 à 1864). Etudes à partir de deux romans de 1862. CHAINTRIER, Pauline, L'instruction judiciaire au XIXe siècle. L'exemple de la Cour d'assises du département de la Vienne.

CHANDES, Pierre, Des parrains corses vus sous le prisme déformant du Temps puis du Monde (1918-1985). CHANTREAU, Aurélie, Les représentations des scandales politiques de l'entre-deux-guerres à travers la presse française.

COIFET, Lucie, La vision des Français sur les civilisations Inca et Mexica au XVIe et au XVIIIe siècles d'après les ouvrages de compilation.

DAIGUEMORTE, Cécile, L'abbaye de Charroux du Moyen-Age à nos jours : entre histoire, mémoire et patrimoine.

DIMOSKI, Vladko, État des lieux d'un nouvel État européen. Identité, images, défis, conflits entre les Balkans et l'Europe, la République de Macédoine 1991-2002.

ERNOULT, Soizic, Tuer et être tué. Les homicides de 1780 à 1790 dans le Présidial de Poitiers.

FERRE, Romuald, De l'alcoolisation à l'alcoolisme. Approche historiographique du XIXe et du XXe siècle. 
GANDOUET, Denis, La Révolution au ras du sol: Les justices de paix dans les campagnes d'Aunis (Charente Inférieure.)

GARREAU, Marie, Société et femmes parisiennes, dans Les Français peints par eux-mêmes. Entre réalité, représentations et opinions.

GAUDIN, Sarah, Au hasard d'une fortune de mer. Étude du naufrage du navire Le Hasard, le 23 novembre 1768 à la côte d'Arvert.

GAUVIN, Damien, L'acheminement de la Slovaquie dans l'Union européenne de 1993 à 2006 : attitudes et évolution de l'opinion publique et des élites politiques face à l'adhésion.

GUERINEAU, Diane, Patrimoine industriel et eau dans la Vienne XIXe siècle XXe siècle.

GUITTON, Frédéric, La publicité de presse. Analyse d'un phénomène de société de 1884 à 1920 à Montréal.

HAMOIR, Clément, Les guides touristiques, un témoignage de l'évolution de la société dans la seconde moitié du xxe siècle : le cas de la Suède et de la Finlande.

HERAULT, Olivier, De ville en ville. Étude de la ville chilienne et péruvienne à travers l'ouvrage d'Amédée François Frézier, Relation du Voyage de la Mer du Sud aux côtes du Chili et du Pérou.

JAMIS, Jennifer, Ecrans et écrits : le cinéma à travers l'Avenir de la Vienne pendant l'entre-deux-guerres. LABBE, Adeline, Du «fleuve Charente » à la mer des Caraïbes : Rochefort, les îles du vent et la Guyane pendant la Paix de Trente ans (1716-1744).

LALOURBECHET DE LEOCOUR, Antoine, Les Noirs dans le regard de Père Augouard. Un missionnaire spiritain à la rencontre des peuples du Gabon et du Congo (1877-1890).

LEBESSON, Thomas, Les vols et les voleurs de biens mobiliers dans le département de la Vienne sous le Second Empire.

LECESVE, Angélique, La commmunauté des chanoines de l'église de Sainte-Radegonde (1780-1790). Étude d'une communauté de clercs séculiers à la veille de la Révolution.

LHOMME, Pierre, S'évader. Les évadés en Poitou à la fin de l'Ancien Régime dans les procédures du greffe criminel du présidial de Poitiers (1759-1790).

MAILLET, Bastine, L'échec de la campagne du parti socialiste lors des élections présidentielles de 2002. MALLET, Nicolas, Les voyageurs vers les Îles de l'Amérique française sur la flotte rochelaise (1770-1788). Migrants temporaires ou définitifs.

MARTINEZ, Marie, Les femmes dans les sociétés populaires de la Vienne pendant la Révolution française. MOINET, Maëva, Discours et regards sur le viol. Étude de cinq procès et trois déclarations de grossesse dans le Poitou de la deuxième moitié du xviIIe siècle.

OBLE, Raphaëlle, Le monde des voleurs au siècle des Lumières, d'après les minutes du greffe du siège royal de Lusignan entre 1700 et 1790.

OLIVIER, Thomas, La perception des minorités arméniennes au sein des Empires persan et ottoman entre le XVIIe siècle et le début du XIXe siècle. A travers les relations de voyages, dictionnaires, encyclopédies et instructions d'ambassades.

PONTONNIER, Clémence, Le milieu scolaire sous la plume des instituteurs. Les écoles publiques primaires du département de la Vienne à travers l'enquête scolaire de 1884. 
PORTIER, Vincent, Peuplement et réseaux dans l'ancien diocèse de Limoges d'après le pouillé de 1315-1317, dir. S. Boisselier.

PRADIGNAC, Nicolas, Les expressions des mécontentements ruraux dans les districts de Châtellerault et de Loudun à l'époque de la Révolution française (1787-1799).

RICOU, Jennifer, La mixité ethnique en Louisiane au XVIIIe siècle.

RUMEAU, Nicolas, La Civilisation des périodiques dans le département de la Vienne de 1848 à 1871.

SHIVA, Homayoun, La Milice et le milicien, l'acceptation d'un service obligatoire en Poitou.

SPECQ, Louise, Aristocrates et bourgeoises. Portrait de femmes dans le Courrier de Paris de Delphine

Girardin. Entre horizons d'attente, représentations et réalités.

STOCH-MICHNA, Katarzyna, La Pologne dans le Mercure de France. Une vision du Royaume de Pologne et des Polonais au travers du Mercure de France entre 1720 et 1745.

WEIL, Florie, Jean-Richard Bloch et L'Effort : de l'ombre à la lumière

\section{Master 2}

\section{4}

BARTHELEMY, Claire, Les transformations de l'image des Troupes de Marine, de la Décolonisation à nos jours. Le régiment d'Infanterie Chars de Marine de Poitiers, témoin de cette évolution.

BERTRAND, Céline, Les empoisonneuses. Regard des experts judiciaires, de la presse et de l'opinion publique sur les empoisonnements en tant que crime féminin, dir. F. CHAUVAUD.

BRIQUET, Elsie, Les bibliothèques populaires de la Vienne sous la IIIe République (1870-1930) (2 volumes), dir. F. AUBOIN et G. BOURGEOIS.

CHARPENTIER, Claire, Les archives de Loudun : historique et contenu du fonds.

COTINAT, Guillaume, Les commissaires de police et leur activité dans la France du XIXe siècle (1791-1914): l'exemple du département de la Vienne.

DAMON, Géraldine, Naissance d'une tétrarchie nobiliaire en Poitou : les vicomtes de Thouars et les seigneurs de Lusignan, Parthenay et Mauléon (IXe-1152) (2 vol.), dir. M. AURELL.

DAMOUR Emmanuelle, Les visites pastorales de Mgr Fabre, évêque de Saint-Denis de la Réunion: 1897-1914, dir. F. VIGIER.

DUPONT, Olivier, La Vienne et le recrutement militaire de l'an VIII à 1808 (2 volumes).

ETSILA, Judicaël, L'insécurité routière dans la presse française de 1945 à nos jours. L'exemple du Libre Poitou-Centre Presse, dir. F. CHAUvAUD.

GREAU, Virginie, Les espaces criminels dans la presse régionale à travers l'exemple de La Nouvelle République du Centre-Ouest de 1945 à nos jours.

MIELCZAREK, Willy, Technique et architecture de la tannerie en France, de la Révolution à la IIIe république 1790-1940.

MOUKTAFI, Sabrine, Textes épigraphiques et manuscrits : la place des inscriptions dans l'histoire ecclésiasthique d'Orderic Vital (1114-1141) : mémoire et biographie, dir. C. TREFFORT et M. AURELL. 
ROMPILLON, Alexandra, L'amphithéâtre de Poitiers de l'antiquité à nos jours. Étude historique d'un patrimoine, dir. J. PÉRET.

VENON, Leslie, L'hippodrome de Cheltenham, miroir de la société britannique.

WEBER Vincent, Les lieux de mémoire de la Nouvelle-France en valeur sur internet, dir. D. GUILLEMET.

YARNYKH, Vera, L'aristocratie en France au sud de la Loire aux IXe-XIe siècles: d'après les sources hagiographiques, dir. M. AURELL.

\section{5}

BENHIDA, Nadia, Les images de la colonisation dans L'Assiette au Beurre. Revue satirique illustrée (1901-1912), dir. F. CHAUVAUD.

BERNARD, Aline, Les juridictions d'exception de l'Occupation et de la Libération, Vienne, CharenteMaritime, Deux-Sèvres, Vendée (1940-1970), dir. F. CHAUVAUD.

BROUARD, Emmanuel, La société rurale en vallée de l'Authion de 1750 à 1914. Les réactions aux crises et aux mutations économiques et sociales dans un environnement contraignant (2 volumes).

FARGE, Arnaud, Adhésion populaire et désobéissance civile sous le Consulat et le Premier Empire.

Perspectives de recherche à travers l'exemple de l'Indre-et-Loire, dir. N. PETITEAU.

FRANÇOIS, Olivier, Les maladies de l'esprit de la gent masculine dans le Dictionnaire encyclopédique des sciences médicales Dechambre, dir. F. CHAUVAUD.

GLATIGNY, Jessica, Les faits divers dans la presse acadienne (L'Evangéline à Moncton) et dans la presse québécoise (La Presse à Montréal). 1945-1982.

MASTORGIO, Laurent, Les Creuzé à Châtellerault (1600-1850). Aspects religieux, dir. J. PÉRET.

PORTAIN, Olivier, La Société des Antiquaires de l'Ouest gardienne de la mémoire du patrimoine bâti dans le Centre Ouest français (1832-1911), dir. F. CHAUVAUD.

WENDLING, Geneviève, Images sociales des « quartiers » à travers les faits divers de la fin du XXe siècle. L'exemple de Centre Presse et des Dernières Nouvelles d'Alsace, dir. F. CHAUVAUD.

\section{6}

CHANCEREL, François, Mobilité, enracinement et ouverture villageoise dans une paroisse de la banlieue de Poitiers : Buxerolles (xviie-début xixe siècle), dir. J. PÉRET.

DEMICHEL, Fanny, Le Vagabondage au XVIIIe siècle dans le ressort du présidial de Poitiers, dir. S. JAHAN.

DIALLO, Dramane, L'Office du Niger depuis sa création à nos jours : la problématique de la gestion de l'espace et des ressources en eau dans la zone de l'office du Niger (Mali).

GROS, Samuel, William Adams ou comment un Européen de XVIIe sècle est-il devenu samurai et pourquoi ?, dir. S. JAHAN.

LIGNER, Florian, Évolution de l'image des pays nordiques dans les œeuvres touristiques début XIXe-début XXIe siècle.

РІснот, Benjamin, Entre France et Espagne. L'usage du témoignage en histoire à travers le récit de vie Lazaro et Consuelo Garcia. 
SEMBEL, Patrick, Discours et mémoires à l'œuvre à l'Ecole Nationale Supérieure des Beaux-Arts de Paris (1934-1968). Un objet d'étude pour une histoire culturelle de l'art.

TALLEUX, Maryline, La limitation des naissances en Poitou (XVIIe- XVIIIe siècles), dir. S. JAHAN.

TRANCHANT, Aurélie, Réflexions sur les noms des voies publiques de Buxerolles, Chauvigny, Montmorillon. Les enjeux de leur dénomination (1859-2006).

TRANCHANT, Laëtitia, Les femmes et l'interrogatoire judiciaire.1844, 1851, 1866 et 1880, dir. F. CHAUVAUD. ZEDIALLAL, Mounia, Insurrections, soulèvements, émeutes, insoumissions : les différentes formes de résistances des habitants de l'Algérie face à l'arrivée et à la colonisation française 1830-1914.

2007

CHARLEUX, Cécile, La représentation de la femme dans le roman canadien : XIXe-XXe siècles.

GANDOUET, Denis, La Révolution au ras du sol: Les justices de paix dans les campagnes d'Aunis (CharenteInférieure).

GIBIER, François, Le second ordre en 1789 : analyse du cahier de doléances de la noblesse de Poitou. GOURINARD, Henri, La noblesse du Royaume latin de Jérusalem (1099-1154) : préliminaires à une étude prosopographique, dir. M. AURELL.

GRIES, Sarah-Jane, Entre martyre et sainteté : la justification des révoltes de Cordoue dans les écrits de saint Euloge : 851-856 (2 vol.),dir. T. DESWARTE, M. AURELL.

GRIMAUD, Noémie, Les Pouvoirs du sexe faible. Symbolismes corporels et perceptions du quotidien féminin dans le Poitou rural (1850-1950).

JEAN, Samuel, Un exemple de justice de proximité au Québec : la cour des commissaires aux petites causes de Saint-Jean-Chrysostome, 1844-1900.

LAVAUR, Ludivine, Relier Poitiers à la Loire et à la mer ? Les projets avortés de la navigation du Clain à la fin du XVIIIe siècle et dans la première moitié du XIXe siècle.

MENDES, Véronique, Voyage en Icarie. De l'histoire contée à l'Histoire mémorable. 1772-2005 La tentation identitaire?, dir. F. CHAUVAUD.

PFENDER, Nicolas, Les missionnaires de la SMEP au Togo de 1929 à 1959.

POUGET, Emmanuel, La politisation des ruraux en Charente-Maritime 1750-1850. Projet scientifique et état des sources. La vie municipale de quelques communautés rurales en Aunis et en Saintonge au XviIle siècle. Premières approches au travers des « actes capitulaires ».

Pousin, Yoan, La prosopographie du personnel parlementaire dans le département de la Vienne (1930-1939).

\section{Thèses}

2007

CIRIER, Aude, De l'Empire à la Commune : essor et mutation d'une nouvelle noblesse. Étude sur le lignage des comtes d'Elci, de la maison Pannochieschi, Toscane, XIIe-XIVe siècle (3 vol.), dir. M. AURELL. 
DEBIAIS, Vincent, Écriture monumentale, écriture publique et écriture personnelle : perceptions, lectures et utilisations des inscriptions dans la communication médiévale (Ouest de la France, XIIIe-XIVe siècle) (2 vol.), dir. M. AURELL.

GABET, Alain, Les structures familiales et les comportements collectifs en Haut-Poitou au XVIIIe siècle (2 vol.), dir. J. PÉRET.

LARIGAUDERIE-BEIJEAUD, Martine, Grandmont. De l'ermitage à la seigneurie ecclésiastique (XIIe-XVIIIe siècles) (4 vol.), dir. J. PÉRET.

LE BRETON de la Bonnelliere, Bertrand, La fiscalité royale sur les denrées et les marchandises dans l'élection de Poitiers au XVIIIe siècle (1714-1791) (2 vol.), dir. C. LECOMTE.

LOUMADINE, Claire, Le rôle de l'hôpital dans la politique de santé à Angoulême pendant la première moitié du XXe siècle, dir. Ch. CHÊNE.

NORDEY-SANCE, Christelle, La seigneurie de Civray à la fin du Moyen-Âge (XIVe-Xve siècles) : crise ou immobilisme? (4 vol), dir. S. BOISSELIER.

VALIN, Claudy, Le conventionnel Joseph-Marie Lequinio : la loi et le salut public (2 vol.), dir. J.-M. AUGUSTIN.

\section{7}

BILLORE, Maïté, Pouvoir et noblesse en Normandie (fin xiie-début xiiie siècle) : de l'autocratie Plantagenêt à la domination capétienne (4 vol.), dir. M. AURELL.

CANTAMESSA, Laurent, Les débuts du syndicalisme en Poitou-Charentes : 1884-1914 (2 vol.), dir. J.-M. AUGUSTIN.

CHAIZE, Thomas, La construction du vaisseau en France au XVIIe siècle, à partir de manuscrits et livres de construction navale (4 vol.), dir. J. PÉRET.

HANCKE-JOLLIOT, Gwendoline, Les femmes nobles languedociennes à l'époque du catharisme (3 vol.), dir. M. AURELL.

Luc, Albert-Michel, Les gens de la mer dans l'île de Ré au XVIIIe siècle (1681-vers 1790). Terriens de la mer, marins d'une terre, dir. J. PÉRET.

YVOREL, Élise, Les enfants de l'ombre : prisons ordinaires et prisons spécifiques pour mineurs en France métropolitaine : la vie quotidienne des jeunes détenus au XXe siècle (4 vol.),dir. F. CHAUVAUD.

2007

AUBERT, Émilie, Les trônes et leurs usages : étude des personnages siégeant dans l'imagerie grecque aux époques archaïque et classique (3 vol.), dir. P. BALLET.

BOUSIGES, Richard, Une famille cévenole au XVIIIe siècle : les Bousiges (2 vol.), dir. D. РОтоN.

DUGUE, Caroline, Les décors en opus sectile de la Gaule romaine : contribution à l'étude du décor romain : Ier siècle av. J.-C.-IVe siècle apr. J.-C. (3 vol.), dir. P. BALLET.

JEANNEAU, Cédric, Le Bas-Poitou du Xe au milieu du XIIIe siècle : organisation de l'espace, affirmation du lignage et évolution des structures de la société (7 vol.), dir. M. AURELL.

KORSIA, Haïm, Jacob Kaplan ou le rabbin de la République, dir. PAUL LÉVY. 
MUSSA, Carlos, Les conflits au Mozambique : la Longue marche vers la Liberté (1930-1992) 2 vol, dir. F. CHAUVAUD.

2007

BAUDRY-BRULET, Eliane, Châteauroux au XVIIIe siècle : ressorts et mutations d'une société urbaine (4 vol.), dir. J. PÉRET.

BETHERY DE LA BROSSE, Arnaud, Entre amour et droit : le lien conjugal dans la pensée juridique moderne (xvie-XXIe siècles) (2 vol.), dir. J.-M. AUGUSTIN.

BOUCHARD, Laurent, Les protestants français face à la monarchie : la difficile recherche d'un modèle d'intégration juridique (1559-1715) (2 vol.), dir. E. Gojosso.

DANAUX, Stéphanie, L'essor du livre illustré au Quebec en relation avec les milieux artistiques et éditoriaux français : 1880-1940 (2 vol.), dir. S. Vernois, L. LAMARCHE.

DELVARE, Anne, Thomas-Louis Bourgeois (1676-1750/51) : contribution à l'histoire de la musique française dans la première moitié du XVIIIe siècle (4 vol.), dir. J. GRIBENSKI.

GUILLON-GUEPIN, Armelle, De l'administration des anciennes villes de commune du Poitou au XVIIIe siècle (3 vol.), dir. C. LECOMTE.

LHERMITTE, Xavier, La construction religieuse à limoges de 1170 à 1273 : enquête sur les monuments du premier siècle de l'architecture gothique (4 vol.), dir. C. ANDRAULT-SCHMIDT.

MOUKARZEL, Pierre, La ville de Beyrouth et son commerce avec les villes marchandes européennes à l'époque Mamelouke (1291-1516) (2 vol.), dir. S. BOISSELIER.

RIGHI, Dominique, Les images dans les manuels de lycée (manuels d'histoire, de français, de philosophie et de langues vivantes publiés de 2000 à 2003) : Les représentations du pouvoirs et des conflits politiques de 1815 à nos jours (3 vol.), dir. F. CHAUVAUD, S. VERNOIS.

\section{INDEX}

Index géographique : Poitiers 